\title{
Economic burden of asthma in Abu Dhabi: a retrospective study
}

This article was published in the following Dove Press journal:

ClinicoEconomics and Outcomes Research

17 October 2014

Number of times this article has been viewed

\author{
Ashraf Alzaabi' \\ Mohammed Alseiari² \\ Bassam Mahboub ${ }^{3}$ \\ 'Respiratory Medicine Division, Zayed \\ Military Hospital, Abu Dhabi, United \\ Arab Emirates; ${ }^{2}$ General Secretariat \\ of Executive Council, Abu Dhabi, \\ United Arab Emirates; ${ }^{3}$ University \\ of Sharjah, Rashid Hospital, Sharjah, \\ United Arab Emirates
}

\begin{abstract}
This study evaluates the direct costs of treating asthma in Abu Dhabi in the United Arab Emirates. Data was compiled for 2011 from health insurance claims covering all medical interventions or treatments coded as asthma. Costs were calculated from a health care perspective. The total direct cost of treating 139,092 asthma patients was estimated to be United Arab Emirates Dirhams (AED) 105 million (US\$29 million), corresponding to around AED 750 per patient per annum. The total cost is principally generated by outpatient visits ( $>$ AED 85 million; $81 \%$ of the total cost). Ten point four percent of patients had made an emergency room visit. The cost per visit seems to be higher during hospital admissions (AED 7,123) compared to outpatient visits and emergency room visits. The direct cost of asthma medications was around AED 33 million ( $31 \%$ of the total cost). The economic burden of asthma in Abu Dhabi is high and the number of emergency visits suggests that the disease is not optimally controlled.
\end{abstract}

Keywords: asthma, cost, Abu Dhabi, UAE, Middle East

\section{Introduction}

Asthma is a common chronic disease characterized by shortness of breath, chest tightness, wheezing, and coughing, ${ }^{1}$ which occurs in all age groups, particularly during childhood. ${ }^{2}$ In 2011, the World Health Organization (WHO) estimated that 235 million people suffered from asthma worldwide. ${ }^{3}$ In the International Study of Asthma and Allergies in Children (ISAAC), 13.8\% of 13-14-year-old children worldwide had asthma at some time in their lives. ${ }^{2}$ The report on the global burden of asthma published by the Global Initiative for Asthma (GINA) estimated that the number of disability-adjusted life years (DALYs) lost due to asthma was estimated at around 15 million DALYs per year, and accounting for around one in every 250 deaths worldwide. ${ }^{4}$

In the United Arab Emirates (UAE), a major survey conducted in 2009 among a randomly selected sample of 1,220 residents of all age groups reported that the prevalence of asthma symptoms fulfilling the definition of the European Community Respiratory Health Survey (ECRHS) screening questionnaire, was 12.1\% (n=146 patients). ${ }^{5}$ Moreover, the Asthma Insights and Realities in UAE (AIR-UAE) study, ${ }^{6}$ a general population survey performed in 2009, reported that two-thirds of the 200 patients identified with asthma reported having sudden severe attacks. Daytime symptoms and nighttime symptoms in the last month were reported by $57.5 \%$ and $35.5 \%$ of subjects, respectively. In terms of the impact of asthma on daily activities, $52.8 \%$ of affected children and $17.1 \%$ of affected adults reported missing school and work in the past year due to their asthma. ${ }^{6}$
Correspondence: Ashraf Alzaabi Respiratory Medicine Division, Zayed Military Hospital, Abu Dhabi, United Arab Emirates Tel +971 506474488

Email ashraf.alzaabi@utoronto.ca 
Given its high prevalence, the severity of associated symptoms, and the considerable impact that it has on patients' lives, asthma imposes a considerable economic burden on health care systems in terms of morbidity and mortality. Two studies from the US reported data on the costs of asthma in 2007. The first $^{7}$ estimated annual direct medical expenditure to be US\$37.2 billion, whereas the second, which also included indirect costs, found the annual total incremental cost of asthma to society to be US\$56 billion..$^{8}$ In Europe, the annual direct cost of asthma has been estimated to be $€ 19.5$ billion. $^{9}$

Two recent studies have been undertaken to determine the costs of asthma in the UAE, using different methodologies, one in Dubai, ${ }^{10}$ based on individual patient-reported health care resource utilization, and one in Abu Dhabi, based on an analysis of insurance reimbursement claims. In the Dubai study, the total direct cost of asthma was estimated to be United Arab Emirates Dirhams (AED) 88 million (US\$24 million).$^{10}$ The current article presents the data obtained in the study performed in Abu Dhabi.

\section{Methods Study design}

This was a retrospective, insurance claims study of the economic burden of asthma in Abu Dhabi. The scope of the analysis was to document the direct costs of treating asthma in Abu Dhabi for all residents (nationals and expatriates), including outpatient treatment costs, hospital admissions, and emergency room visits. Costs were calculated from a health care perspective.

The study used data provided by the National Health Insurance of Abu Dhabi, which covers $98 \%$ of the total population of the emirate and documents all health care resource utilization in an electronic database (e-claims). It is possible to cross the data in the insurance claims database with parallel information provided by health care providers.

\section{Study population}

At the end of 2011, the Abu Dhabi Chamber of Commerce and Industry estimated that the emirate had a total population of around 2.9 million inhabitants. ${ }^{11}$ The target population of the present study consisted of all patients who visited a public or private health care provider because of asthma. Asthma patients recorded in the database of the Health Authority of Abu Dhabi (HAAD) were identified according to the WHO's 9th International Classification of Disease (ICD-9). ${ }^{12}$ This makes it relatively easy to identify visits related to asthma with a high degree of accuracy.
The study sample was extracted from the database provided by the HAAD. All patients of all ages who visited a health care provider for asthma during 2011 were included in this analysis.

Since the access to patient data is restricted to authorized personnel only, we specifically asked the HAAD to perform an analysis of their data to estimate the cost of asthma e-claims in 2011. Data related to physician consultations, hospital admissions and emergency room visits, medication, and paraclinical procedures were extracted from the HAAD database. Access to the characteristics of the study population such as demographics, history of asthma, severity of the disease, and presence of comorbidities was not possible.

\section{Identification and allocation of costs}

Basic pricing of health services in Abu Dhabi is regulated at the national level by the HAAD either for public or private hospitals. With respect to this price reference, health care providers and insurance companies negotiate the real costs that they will finally apply.

In Abu Dhabi, when a patient uses a health care service, the health care provider charges the subscribed insurance company, which reimburses the fees based on claims submissions. Consequently, costs are eligible for reimbursement, which vary from patient to patient according to the level of their health insurance coverage. In Abu Dhabi, three health insurance programs exist. These consist of: 1) the Basic program, which covers $45 \%$ of the population; 2) the Enhanced program, which covers $39 \%$ of the population; and 3 ) the Thiqa program which covers $16 \%$ of the population and which is exclusively used by nationals. The Basic program is subsidized by the Abu Dhabi government. Employers pay a fixed fee for employees who have low salaries in order to offer them a basic health care insurance coverage provided by a third-party authority, called Daman. The government covers any costs above what the employer has paid. The Enhanced program is a program in which employers buy a "package" of health care coverage for their employees. Costs above the chosen "package" are paid by the patients themselves. The Thiqa program is a government program managed by Daman, in which treatment costs are covered in totality when the patients receive health care in government hospitals and partially when care is provided in private hospitals.

In the interests of estimating the real costs related to the treatment of asthma in Abu Dhabi, costs were analyzed based on claims submission invoices collected by the HAAD from the insurance companies, together with costs applied by health care providers. The sum of these different elements in the e-claims 
allows us to estimate, as closely as possible, real expenditure for the treatment of asthma from a health care perspective.

Costs are reported in Dirhams (AEDs) based on 2011 tariffs. No discounting was attributed.

\section{Statistical analysis}

For descriptive statistics, data were expressed as mean \pm standard deviation, or as percentages for categorical variables.

\section{Ethics}

The study was performed in the framework of relevant international and national legislation. This study was approved by the Scientific Committee of the Zayed Military Hospital.

\section{Role of the funding source}

This study was initiated by the Respiratory Care Unit and Sleep Disorder Centre of the Zayed Military Hospital in UAE. This study received an unrestricted research grant from GlaxoSmithKline. A scientific committee was appointed to advise on the design, implementation, and interpretation of the study. This committee had full access to all data from the study. Committee members did not receive any financial compensation in return for their participation. The corresponding author had final responsibility for the decision to submit the manuscript for publication.

\section{Results}

\section{Study population}

From the data held on file by the HAAD for 2011, a total of 139,092 subjects covered by health insurance used health care facilities and received treatment for asthma in Abu Dhabi and these represent the study population.

\section{Costs incurred during inpatient and outpatient visits}

The study population made a total of 350,028 visits to medical facilities for management of their asthma. Asthma was managed during outpatient visits $(311,719 ; 89.0 \%)$ in the majority of cases, followed by direct emergency room visits $(36,257 ; 10.4 \%)$, overnight hospitalization $(1,231 ; 0.4 \%)$, and overnight hospitalization following an emergency room visit $(821 ; 0.2 \%)$.

The total cost related to asthma treatment in 2011 in the UAE was estimated at around AED 105 million, approximately US\$29 million. This total cost was incurred principally during outpatient visits (over AED 85 million; $81 \%$ of the total cost). In terms of unit costs, overnight hospitalizations were the most expensive resource item (AED 7,123 per admission) followed by hospital admissions and emergency room visits (AED 5,689 per admission), outpatient visits (AED 274 per visit), and direct emergency room visits (AED 167 per admission).

\section{Costs by resource item}

The contributions to total direct medical costs were assessed for medication acquisition costs (stratified into controller medication and rescue medication), procedures (including laboratory tests, spirometry, and radiology), and hospital overheads (including personnel, administration costs, board, and lodging). The direct cost of asthma medications was estimated to be AED 32 million, representing 31\% of the total direct costs (Table 1).

Acquisition of controller medication was ten times more costly than acquisition of rescue medication. Procedures accounted for AED 7 million, with the remaining AED 65 million corresponding to hospital overheads. The majority of costs associated with medication acquisition (95.8\%) and of procedures (89.6\%) was generated during outpatient visits.

Asthma treatment costs were also evaluated separately for each resource used (medication, emergency visits with or without hospitalization, hospitalization alone, outpatient visits, and procedures) during the management of asthma in Abu Dhabi (Figure 1). The direct cost of outpatient man-

Table I Costs of asthma drugs, related procedures, and hospital overheads according to the type of visit in Abu Dhabi in $201 \mathrm{I}$

\begin{tabular}{|c|c|c|c|c|c|c|}
\hline & $\begin{array}{l}\text { Outpatient } \\
\text { visits }\end{array}$ & $\begin{array}{l}\text { Hospital } \\
\text { admissions }\end{array}$ & ER visits & $\begin{array}{l}\text { Hospital } \\
\text { admissions } \\
\text { and ER visits }\end{array}$ & $\begin{array}{l}\text { Total } \\
\text { cost }\end{array}$ & $(\% *)$ \\
\hline Medication & $30,997,312$ & 17,342 & $|, 266| 5 \mid$, & 60,722 & $32,341,527$ & $30.9 \%$ \\
\hline Controller medication & $28,180,393$ & 14,327 & 950,172 & 49,537 & $29,194,429$ & $27.9 \%$ \\
\hline Rescue medication (SABAs) & $2,816,919$ & 3,015 & 315,979 & 11,185 & $3,147,098$ & $3.0 \%$ \\
\hline Procedures & $6,397,323$ & 40,747 & 499,817 & 202,315 & $7, \mid 40,201$ & $6.8 \%$ \\
\hline Hospital overheads & $47,922,405$ & $8,7 \mid 0,406$ & $4,276,28 I$ & $4,407,851$ & $65,316,944$ & $62.3 \%$ \\
\hline
\end{tabular}

Notes: *For each column, the denominator is the respective total costs mentioned in Table I. The numbers in bold represent the higher and lower values. Costs are presented in United Arab Emirates Dirhams.

Abbreviations: ER, emergency room; SABAs, short-acting beta agonists. 


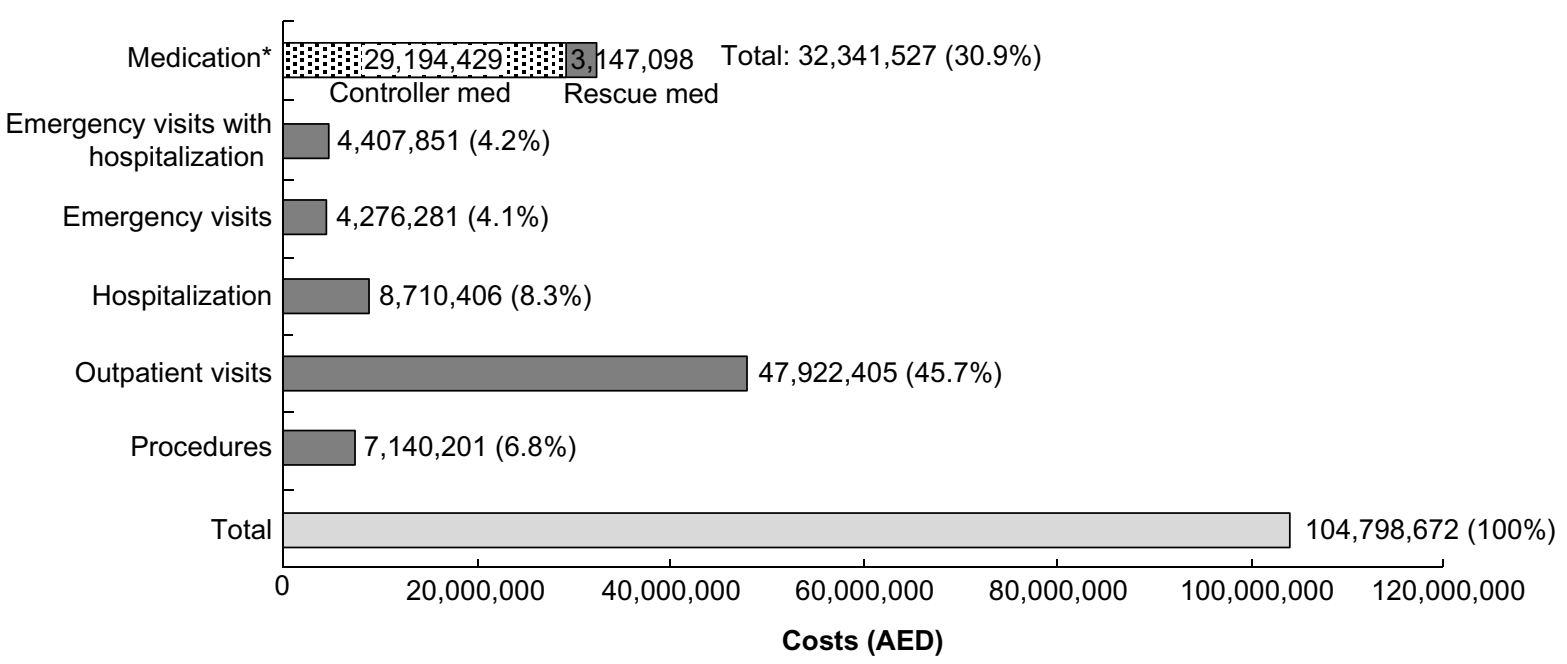

Figure I Distribution of asthma treatment costs according to the resource used in Abu Dhabi for the management of asthma.

Note: Categories are mutually exclusive. *The dotted shading designates controller med (29,194,429 AED), and the dark grey shading designates rescue med (3,147,098 AED). Abbreviations: AED, United Arab Emirates Dirhams; med, medication.

agement represented the highest expenditure $(45.7 \%$ of the total cost).

\section{Discussion}

This study documented the direct costs of asthma treatment according to health care resources used in the emirate of Abu Dhabi, based on national health insurance claims data for 139,092 subjects with asthma. To our knowledge, the figures from this new investigation are the first to evaluate the economic burden of asthma in Abu Dhabi using exhaustive data on health care resource utilization and related financial data. Not surprisingly, asthma treatment causes a substantial financial burden on health care with an estimated total direct cost of asthma treatment of around AED 105 million (US\$29m). This represents a per capita cost of approximately AED 750 (US\$200/€160).

The per capita costs in our study can be compared with those documented in other health systems. A study from Europe, which estimated the prevalence and the cost of asthma in five countries in 2006, revealed that the direct cost per asthma patient was around $€ 280 .{ }^{13}$ Similarly, a study from Canada reported that, in 2006, per capita direct cost of asthma treatment was approximately US $\$ 300 .{ }^{14}$ This suggests that in Abu Dhabi the per capita direct costs are about half to twothirds of those of European and North American levels. This difference may be explained in part by the lower unit costs of hospitalization and of certain medications and in part by a higher proportion of patients managed in the community. Little data are available on the cost of other diseases in the UAE. However, the per capita annual direct cost of asthma seems to be approximately ten times lower than the total annual direct cost of diabetes mellitus without complications, which was estimated at AED 5,900 (US\$1,605) per capita in a study performed in 2005. ${ }^{15}$

The cost of asthma in our study is primarily borne by outpatient care, which contributes approximately $80 \%$ of direct costs, suggesting that the management of asthma in Abu Dhabi is performed in the majority of cases outside hospitals. The contribution of outpatient care to overall costs seems to be higher compared to that reported in a large study performed in Europe in 2002, which estimated that around half (48\%; $€ 3.8$ billion) of the annual direct cost of treating asthma accrued through outpatient care. ${ }^{16}$ Nonetheless, we found that around $20 \%$ of total asthma cost in Abu Dhabi was associated with hospital admission and emergency room visits, which are potentially related to poor asthma control and occurrence of exacerbations; the number of emergency visits was also considerable (over 36,000 visits). This suggests that the management of asthma in Abu Dhabi may not be fully optimal and that better disease control needs to be achieved. This strategy can potentially be a cost-saving option, as shown in other studies from the US and Europe. ${ }^{17,18}$ In terms of individual resource components, physician consultations accounted for $46 \%$ of total direct costs and the cost of medication accounted for $31 \%$.

Based on the national health insurance database, the number of individuals in Abu Dhabi who were treated for asthma in 2011 was estimated to be 139,092 . With respect to the total population of Abu Dhabi (2.9 million habitants), this corresponds to a crude prevalence of around $4.8 \%$. This percentage is much lower than the expected prevalence of asthma and indeed of that determined in a population-wide 
epidemiological study performed in the UAE, ${ }^{5}$ which estimated that $12.1 \%$ of the population fulfilled the ECRHS asthma definition. This suggests that an important group of potential asthma sufferers is not covered by the national health insurance, not diagnosed, or not treated.

An interesting aspect of this study is to compare our findings, obtained through an exhaustive analysis of the national insurance claims database, with another study performed around the same time in the neighboring emirate of Dubai, ${ }^{10}$ which has a very similar population and health care system. In the latter study, data were collected from patient questionnaires. The Dubai study reports a lower proportion of cost attributed to consultations and medications and a higher proportion attributed to hospitalization. Although it cannot be excluded that hospitalizations for asthma are more frequent in Dubai than in Abu Dhabi, recall bias on the part of participants, who are more likely to remember hospitalizations than consultations may also contribute to this difference. Similarly, the lower overall per capita costs identified in the Dubai study may reflect underreporting.

Compilation of data from the national insurance claims database has a number of advantages. Under this system, data are compiled for all health care providers and costs can be audited and reconciled between providers and insurers. Consequently, data are subject to independent scrutiny and may provide a very realistic view of the direct costs involved in treating asthma. However, inevitably, some insurance claims fail to be integrated into the electronic database, notably data from small clinics processing very small amounts of data. The HAAD estimate that these could reduce claims coverage to between $85 \%$ and $90 \%$ of the true total. Consequently, our cost estimates here may be a little conservative.

Nonetheless, this study has a number of limitations. In particular, only direct costs were measured. The inclusion of other costs, including nondirect medical costs (such as transport to hospitals), indirect costs (such as days lost from work and school), and intangibles (such as quality of life and social consequences) is likely to result in an even greater total cost burden of asthma, with indirect costs potentially outweighing direct costs. ${ }^{19}$ For example, the AIR-UAE study performed in the UAE in 2008 revealed that $21 \%$ of subjects with asthma had reported days lost from school or work in the past year due to their asthma. In the European AIR study, the cost of lost productivity because of asthma was around $€ 10$ billion. ${ }^{16}$ In addition, the insurance claims database used in our study did not contain information on demographics or clinical characteristics of the study population such as level of severity or asthma control, which have been demonstrated elsewhere to be important determinants of cost. ${ }^{19,20}$

Our findings need to be completed by future studies assessing the use of health care resources in naturalistic treatment conditions, such as epidemiological studies involving physicians and patients, which will be able to collect cost data from individual patients and relate resource consumption to demographic and clinical characteristics of the patient, such as asthma severity and control. This would allow a much more refined analysis of resource consumption than aggregate data obtained from insurance claims databases. Our data, together with findings from individual patient surveys, will be of use to guide clinicians in managing asthma better and help policymakers plan and allocate national expenditures for asthma treatment in a more rational way.

\section{Conclusion}

This study revealed that the annual direct cost of asthma in Abu Dhabi is substantial and causes an important economic burden. Treatment of asthma in outpatient visits represents the most important source of direct medical expenditure. However, emergency visits and hospitalizations are significant, suggesting that the control of asthma is suboptimal. The situation could be improved by encouraging early diagnosis of the disease, by use of effective controller medication, and by increasing awareness and information among the general population; such measures could generate significant health care resource savings in the long-term.

\section{Acknowledgment}

The authors would like to thank Foxymed (Paris, France), a medical communication agency, for editorial assistance and contribution for the preparation of this article.

\section{Disclosure}

Ashraf Alzaabi has received honoraria from GlaxoSmithKline Laboratories for this study. Mohammed Alseiari and Bassam Mahboub have no conflicts of interest to declare in this work.

\section{References}

1. Calverley P, Pauwels RA, Jones PW, Anderson JA, Vestbos J. The severity of airways obstruction as a determinant of treatment response in COPD. Int J Chron Obstruct Pulmon Dis. 2006;1(3):209-218.

2. Pearce N, Ait-Khaled N, Beasley R, et al; ISAAC Phase Three Study Group. Worldwide trends in the prevalence of asthma symptoms: Phase Three of the International Study of Asthma and Allergies in Childhood (ISAAC). Thorax. 2007;62(9):758-766.

3. World Health Organization. Asthma fact sheet [updated Nov 2013] Available from: http://www.who.int/mediacentre/factsheets/fs307/en/. Accessed March 17, 2014. 
4. Masoli M, Fabian D, Holt S, Beasley R. The global burden of asthma: executive summary of the GINA Dissemination Committee report. Allergy. 2004;59(5):469-478.

5. Mahboub BH, Al-Hammadi S, Rafique M, et al. Population prevalence of asthma and its determinants based on European Community Respiratory Health Survey in the United Arab Emirates. BMC Pulm Med. 2012;12:4

6. Mahboub BH, Santhakumar S, Soriano JB, Pawankar R. Asthma insights and reality in the United Arab Emirates. Ann Thorac Med. 2010;5(4):217-221.

7. Kamble $\mathrm{S}$, Bharmal M. Incremental direct expenditure of treating asthma in the United States. J Asthma. 2009;46(1):73-80.

8. Barnett SB, Nurmagambetov TA. Costs of asthma in the United States: 2002-2007. J Allergy Clin Immunol. 2011;127(1):145-152.

9. European Respiratory Society. The economic burden of lung disease. Available from: http://www.erswhitebook.org/chapters/the-economicburden-of-lung-disease/. Accessed September 1, 2014.

10. Mahboub BH, Shendi F, Safarini BK, AbdulAziz MH, Mustafa GM, Prakash V. Cost of asthma in Dubai, United Arab Emirates (UAE). J Pulmon Resp Med. 2013;3:146.

11. Abu Dhabi Chamber of Commerce and Industry. Annual Report 2012. Available from: http://www.abudhabichamber.ae/PublicationsEnglish/ Doc-29-7-2013-133651.pdf. Accessed September 1, 2014.

12. The International Classification of Diseases, 9th Revision (ICD-9) [webpage on the Internet]. Atlanta: Centers for Disease Control and Prevention; 2014 [last updated September 1, 2009]. Available from: http://www.cdc.gov/nchs/icd/icd9.htm. Accessed May 16th, 2014.
13. Demoly P, Paggiaro P, Plaza V, et al. Prevalence of asthma control among adults in France, Germany, Italy, Spain and the UK. Eur Respir Rev. 2009;18(112):105-112.

14. Sadatsafavi M, Lynd L, Marra C, et al. Direct health care costs associated with asthma in British Columbia. Can Respir J. 2010;17(2):74-80.

15. Al-Maskari F, El-Sadig M, Nagelkerke N. Assessment of the direct medical costs of diabetes mellitus and its complications in the United Arab Emirates. BMC Public Health. 2010;10:679.

16. Gibson GJ, Loddenkemper R, Sibille Y, Lundbäck B, editors. European Lung White Book. Sheffield, UK: European Respiratory Society; 2003 .

17. Centers for Disease Control and Prevention. National Health Statistics Report: Asthma Prevalence, Health Care Use, and Mortality: United States, 2005-2009. Hyattsville, MD: US Department of Health and Human Services. January 12, 2011; No 32.

18. Rabe KF, Vermeire PA, Soriano JB, Maier WC. Clinical management of asthma in 1999: the Asthma Insights and Reality in Europe (AIRE) study. Eur Respir J. 2000;16(5):802-807.

19. Accordini S, Bugiani M, Arossa W, et al. Poor control increases the economic cost of asthma. A multicentre population-based study. Int Arch Allergy Immunol. 2006;141(2):189-198.

20. Global Initiative for Asthma. Global Strategy for Asthma Management and Prevention [Updated May 6, 2014]. Available from: http://www. ginasthma.org/local/uploads/files/GINA_Report_2014_Aug12.pdf. Accessed September 2, 2014.
ClinicoEconomics and Outcomes Research

\section{Publish your work in this journal}

ClinicoEconomics \& Outcomes Research is an international, peerreviewed open-access journal focusing on Health Technology Assessment, Pharmacoeconomics and Outcomes Research in the areas of diagnosis, medical devices, and clinical, surgical and pharmacological intervention. The economic impact of health policy and health systems

\section{Dovepress}

organization also constitute important areas of coverage. The manuscript management system is completely online and includes a very quick and fair peer-review system, which is all easy to use. Visit $\mathrm{http}: / /$ www.dovepress.com/testimonials.php to read real quotes from published authors. 